\title{
The Influences of New Media on the Young Students and Its Countermeasures
}

\author{
Meng Shao \\ Nanyang Institute of Technology, Nanyang, Henan Province, China
}

Keywords: New Media, Young Students, Positive Impact, Negative Impact, Countermeasures.

\begin{abstract}
New media, which relies on digital, communication and Internet technology to provide information services to the public, plays an increasingly important role in enhancing the efficiency of information acquisition and exchange. However, for young students, on the one hand, they accept the positive impact of the arrival of new media; on the other hand, their learning style, the way of thinking and value orientation has also been impacted unprecedentedly. In this paper, the characteristics of new media are expounded first, and then the influence of the new media on the young students is discussed from both sides. Finally, the corresponding countermeasures are given from various aspects. By this, hope to avoid the negative impact of new media and guide the healthy growth of young students.
\end{abstract}

\section{Introduction}

With the rapid development of new media, China's economic society is undergoing positive and profound changes; meanwhile, young students are also facing unprecedented shocks and challenges. This is because the society is full of temptations and obstacles in the new media era, in which differences, contradictions and conflicts have become (or are becoming) inherent elements. To ensure healthy growth and social harmony and stability, Young students should take the initiative to think about the negative impact to learning methods, ways of thinking and value brought by new media, while take the advantage brought by it.

\section{Analysis of New Media's Characteristics}

New media, a new media form supported by new technology, is called "the fifth media", which plays an important role in access and communication. Therefore, new media has largely changed the young students' lifestyles, thinking patterns, communication modes and values, and its specific characteristics are reflected in the following aspects:

Multi-dimensional. Due to the emergence of new media, the physical boundaries of original media form are gradually disappearing, and the same world spread, cross-border communication are becoming the main features of information dissemination in new media era. Information, dynamic or static, or with text, voice, images, video are all able to achieve multi-media communication with same carrier. In addition, with the help of new media, the spread of information has crossed the geographical limitations, which enable a broader space-time sharing of information [1]. It can be said that in the new media field, the three-dimensional space perception is taking shape, both individual users and social organizations are able to show three-dimensional imaging feel, which shows the trend of specific and three-dimensional information.

Users' experience. For individuals, the depth of experience with new media is a very important communication feature. In this field, through the point-to-point personality services, so that experience to produce a more direct sense of immersion, to meet people's social demands. For example, in the WeChat settings, you can choose their own favorite topics, users, public numbers and other concerns, and in its private interface, the user can see the information they need. This will not only save the time cost of screening information, but also to enhance the sharing and exchange of fun. 
Although the concept of personalized service is not derived from the new media, but the new media has this idea has been specific, and strengthen the dependence of new media users and attribution, the user's control of the information reached an unprecedented level.

Wide spread. In the new media field, whether through the news website, or the mobile terminal access, you can express views and attitudes with the help of information links, perspective events background information and related topic report from those channels. It can be said that the emergence of new media enables news information become very rich in content, exceptionally convenient in accessing, extremely wide in spreading, and all of these are difficult to achieve in traditional media system. While expanding the scope of information dissemination, at the same time, new media also greatly shorten the transmission time of information, which particularly shows real-time characteristic. With the help of new media, all information can be transmitted to any information receiving terminal in very short time, breaking constraints of time and space and eliminating barriers and intervals among all exchange accesses.

Strong interaction. The transmission of information in traditional media is one-way, while in new media is two-way. Information publisher or recipient can also become information publishers, transmitters and commentators, these "members" of can interact and exchange with new media platform [2]. This brilliant interaction is the traditional media lack of. In the new media field, with the mobile Internet and instant messaging tools, any individual can share, delivery and comment information with any other in the world. What's more, the cost of new media information dissemination is very low, which really enable equality for public in the information consumption relationship; achieve the unity of subject and object.

\section{Impacts of New Media on the Young Students}

New media has had a tremendous impact on young students' learning, life and work. New media expands the scope and vision of young students' social interaction and enhances the ability of acquiring knowledge and comprehensive quality, which have great significance in promoting the process of socialization and realizing the "comprehensive development of human beings". However, there are some young students failed to take the initiative to filter out the wrong thoughts, ideas and behavioral paradigm in new media field, resulting in ideological and behavioral confusion, which are not conducive to young students' growth.

Positive impact. New media technology broke through the limitations of time and space, greatly broadening the range of young students' interpersonal relationships, while breadth and width of interaction in traditional society is susceptible to limitation of geographical space. With the arrival of new media era, unprecedented changes have been brought to ways of communication, under the help of convenient network communication technology, virtual network space. A more open, independent, convenient, flexible, diverse and fast communication mode is attracting many young students by its unique advantage. Rational use of these new media, it is great beneficial for young people to expand interpersonal space, broaden horizons and accumulate rich social network resources.

Compare to traditional media, new media build a more open, independent learning platform for young students. Firstly, young students can independently collect needed knowledge and information quickly and accurately through the massive knowledge platform built by new media. Secondly, in order to enrich knowledge and skills, young students can join a different network of learning groups to communicate with other members online, based on personal needs, and online education has become a new model of learning. Thus, an open exchange learning platform built by new media has optimized channels and capacity of accessing knowledge for young students, greatly broadened the breadth and depth of accessing knowledge to meet the knowledge thirst and stimulate creativity of young students.

A network platform built by new media enables young students to achieve their own socialization continuously. New media presents a colorful world to young people and form a cultural environment gradually that affects growth and socialization of young students. New media, with its distinctive openness, freedom, interaction, equality and other characteristics, meet young students' social expression of personality, advocating freedom, pursuit of equality and other. They can use the forum, 
We Chat, Weibo and other platform to express views on interested events anytime and anywhere, can participate in the interaction process equally to learn and shape citizenship, in order to lay a better foundation for developing democratic idea. Therefore, young people are much more willing to accept and use new media, to achieve and complete their own socialization in the network society created by new media.

Negative impact. In the new media field, information spread at an unprecedented speed. For young students, they are not concerned about all information in transmission, due to the reluctance to analyze, classify and identify information in detail, part of young students give too much attention to meet the self-desire, give a high rating to their own "talent", even produce an illusion that they are better in new media world than in reality. In this way, they will promote themselves more actively and efficiently in the new media field, hoping to find a sense of self-confirmation. In fact, this is the performance of rational thinking dilution, and weak identification of information.

In the new media field, information transmission with the Internet and other media is very common and non-controversial. However, it is undeniable that new media technology usually replaces in-depth report to simple and fast news. For young students, they prefer to accept the news from the new media due to reliance, think with media trend gradually, and lose the ability of independent thinking over time. In addition, because of the "decentralized" features of new media, information is plentiful but difficult to distinguish, and this can easily lead thinking chaos, even loss of will and self to young students, which are extremely unfavorable for young students to from correct outlook of world, life and values, even lead the emergence of national nihilism.

In the new media field, the key carrier of information dissemination is virtual mobile network space, and the existence of virtual space is meet the individual information demands to greatest extend from different channels and different types. With the help of new media technology, network information disseminate rapidly, however, not all the disseminated information withstand security [3]. The reasons leading to such a problem are that anonymity of information publishers, diversity and uncertainty of information disseminators and difficulty of pursuing the fault of information dissemination. All these reason fostered some people to touch the public moral bottom line constantly. Once young students are affected by it, they are easy to confuse right and wrong.

Interpersonal communication in new media field is very different from traditional one. In new media, the features of security and hidden can meet people's psychological fit at a large extent -want to express ideas while worry about danger. In this way, young students will have a strong sense of dependence on new media. But when they return from the virtual world of new media to real world, they are possible to treat people and things in a cold gesture. For example, some young students dare to speak on the network, can clearly express their own ideas, but in real life they perform in a very cold way. There is a survey shows that the stronger the dependence on new media, the greater probability that young students are out of touch with real world, the more their interpersonal and communication skills will be affected.

\section{Countermeasures to the Young Students' Work in New Media}

New media, just as a "double-edged sword", has both positive and negative impacts to young students. In order to enhance its positive impact and eliminate its negative impact, except active guidance and education to students, school should actively build a linkage system with campus culture, construct a media system which positively affect young students, and set up a platform to enhance young students' literacy to network, in order to train and guide young students to healthy growth [4].

To reconstruct the linkage system connecting to campus culture. More and more experience shows that building a campus network culture conforming to the new media is an important platform to enhance network media quality of young students, and also an extension of the new media and the Internet. Therefore, in the actual work, more attention should be paid to construct and plan the future development of campus culture. At the same time, we should use many carriers in new media field to carry out related, positive cultural activities which cater to the interest of young students. 
To build a media system which positively affects young students. Building the media system which actively affects young students is the sacred mission assigned to school in new media age, and also requirements raised by the party to young students' work. Therefore, the school should keep up with trend of times, combined with the characteristics of young students' growth, with the new media platform, exploring innovative ways of work continuously. In addition, establish own new media system to seize new position in young students' education, on behalf of blog, Weibo, We Chat, QQ, Fetion and so on, is also required. For example, set proprietary channels to strengthen guidance and service about employment or entrepreneurship to young students on the platform of weibo, WeChat and other platforms.

To set up a platform to enhance network media literacy of young students. In new media era, with a certain degree of network media literacy is the basic quality of young students. Schools should actively set up a platform to enhance the network media literacy of young students. First, colleges and universities should set up courses related to network media literacy education, such as adding such content in the ideological class, setting up media training courses, or media literacy related lectures, so that each student have the opportunity to accept the network media literacy education. Second, set up relevant knowledge contest through some welcome ways, so that young students accept the network media education in a more relaxed and pleasant environment. Third, make full use of campus media resources, like newspapers and radio, organize young students to participate in media practice in various forms, through the combination of theory and practice, so that the network media literacy education can make great difference.

\section{Conclusions}

In new media field, information dissemination is virtual and uncontrollable, if not avoided and contained, it is easy to increase disharmony and instability to society. The young students' mind is in the mature stage, they have to realize themselves are both user and main beneficiaries in the process of using new media. Young students should recognize he negative impact, while accepting convenience and content richness brought by new media. Due to this, young students should avoid thinking chaos, extreme speech and weak interpersonal relationship through self-restraint and external constraints, to ensure that young students can continuously learn from new media, and constantly abandon the bad information, in order to achieve own growth and social stability and development.

\section{References}

[1] Hao Liu \& Wan-yin Li. The Necessity of Cultivating the Tolerance of Young Students in the New Media [J]. Journal of Huaibei Normal University (Philosophy and Social Sciences), 2015 (8): 163-165.

[2] Xiao-na Zheng \& Xue Zhang. Strategies for Constructing New Media's Influence on Young Students [J]. Journal of Shenyang Agricultural University (Social Sciences Edition), 2014 (9): 557-559.

[3] Chuan-zhong Wang. The Influences of New Media on the Life, Study and Thought of Young Students [J]. Theoretical Front In Higher Education, 2015 (2): 40-41.

[4] Mei-xiang Wang. Analysis of the Effect of New Media on Young Students [J]. New Media Research, 2016 (1): 86-87. 